TITLE:

\title{
Limit analysis for stiffened eight- bolted base plate connections in EHV transmission towers
}

\section{AUTHOR(S):}

LIU, Hongjun; LIU, Yazhou; LI, Zhengliang; HUANG, Haibin

\section{CITATION:}

LIU, Hongjun ...[et al]. Limit analysis for stiffened eight-bolted base plate connections in EHV transmission towers. Japan-China Workshop on Analysis and Optimization of Largescale Structures 2018: 1-2: A01.

ISSUE DATE:

2018-05-14

URL:

http://hdl.handle.net/2433/231234

RIGHT: 


\section{Limit analysis for stiffened eight-bolted baseplate connections in EHV transmission towers}

Hongjun $\mathrm{LIU}^{1,2^{*}}$, Yazhou LIU ${ }^{1,2}$, Zhengliang LI ${ }^{1,2}$, Haibin HUANG ${ }^{1,2}$

${ }^{1}$ School of Civil Engineering, Chongqing University, Chongqing 400045, China

${ }^{2}$ Key Laboratory of New Technology for Construction of Cities in Mountain Area

(Chongqing University), Ministry of Education, Chongqing 400045, China E-mail address: LHJ20040308@126.com (H. Liu)

Keywords: EHV transmission tower, Base plate, Stiffener, Yield-line theory, Uplift load.

\section{Introduction}

With the increasing application of EHV transmission towers in the Chinese electronic industry, eight-bolted base plate (EBBP) connections have been utilized instead of the conventional four-bolted base plate (FBBP) connections in order to withstand the increasing magnitude of uplift load. Currently, there are no industry standards that provide specific requirements for the analysis of EBBP connections in latticed steel transmission structures. The design method of FBBP connections is directly implemented to EBBP connections, which is unrealistic [1]. Thus, it is critical to conduct further investigations on the mechanical properties of EBBP connections.

This study is devoted to developing a theoretical analysis method based on yieldline theory to predict the ultimate strength of EBBP connections under uplift load. A total of 15 specimens were tested. According to the test results, high attentions have been paid to the effects of stiffeners for rigid EBBP connections. Four yield-line models for rigid EBBP connections, considering the contributions of stiffeners, are presented:

Model 1 is applicable to such a connection that incorporates flexible anchor bolts and flexible stiffeners. The base plate, stiffeners and anchor bolts will simultaneously undergo plastic deformation when the connection attains its ultimate strength. Including all these contributions, the ultimate load for Model is derived as follows:

$$
T=\left[\left(10-\sqrt{2} \frac{d}{S}\right) f_{y} t^{2}+\frac{2 f_{y, s} h_{s}^{2} t_{s}}{S}+\frac{2 \sqrt{2}}{3} \frac{f_{y, b} d^{3}}{S}\right] /\left(1+\frac{\sqrt{2}}{3 \pi} \frac{d}{S}\right)
$$

where $f_{y, s}, h_{s}, t_{s}$ are the yield strength, depth and thickness of stiffeners, $S$ is the distance between the bolt hole centre and the centre line of the adjacent boot plate, $d$ is the bolt diameter, $f_{y, b}$ is the yield strength of bolts.

Model 2 is applicable to such a connection that incorporates rigid anchor bolts and flexible stiffeners. Plastic deformation will occurs solely in base plates and stiffeners when the connection attains its ultimate strength, and the ultimate load is given as

$$
T=\left(17.366-3.311 \frac{d}{S}\right) f_{y} t^{2}+2 f_{y, s} t_{s}^{2}+f_{y, s} t_{s} \frac{h_{s}^{2}}{S}
$$

Model 3 is applicable to connections with rigid stiffeners, and the stiffeners are thin but of large depth. The plastic deformation occurs in base plate and the intersection between stiffeners and base plate solely. The ultimate load is

$$
T=\left(22-4.828 \frac{d}{S}\right) f_{y} t^{2}+4 f_{y, s} t_{s}^{2}
$$


Model 4 is applicable to connections with rigid stiffeners, and the stiffeners are thick but of small depth. The plastic deformation occurs only in base plate . The ultimate load is

$$
T=\left(24-4.828 \frac{d}{S}\right) f_{y} t^{2}
$$

For the flexible connections, limited by the test results, only one plastic mechanism were observed with plastic deformation occurring only in base plate. And one yield-line model is proposed.

Compared with the results of design models in the corresponding code, the present models for rigid connections appear to be more accurate. From the parametric analysis, it is implied that the presence of the stiffeners can efficiently increase the strength of the rigid connection and when the stiffeners are of sufficient stiffness, this increasing effect disappears.

The presented model for flexible EBBP connections has also been verified by experimental results, and it appears that this model gives a more accurate prediction for flexible connections with thinner base plate. More accuracy has been demonstrated in comparison with results from the corresponding code as well. According to the parametric analysis, the strength of flexible connections can be improved by optimize the location of anchor bolts. Since the behavior of connections with flexible edge stiffeners (or thick base plate) is not included herein, further investigations need to be conducted on that topic.

\section{Acknowledgements}

The research reported herein was sponsored by the Natural Science Foundation of China (No. 51508054) and Chongqing Basic and Frontier Research Project (cstc2015jcyjA00041). The writers would like to pay their gratitude to all the sponsors for their financial aid.

\section{References}

[1] DT/T5154-2012. Technical code for design of tower and pole structures of overhead transmission line. Beijing: China Planning Press; 2012[In Chinese]. 